\title{
Satisfacción laboral y clima organizacional en funcionarios de atención primaria de salud de una comuna en Chile
}

\section{Job satisfaction and organizational climate in primary health care staff of a commune in Chile}

\author{
Mauricio Araya Salgado \\ Kinesiólogo. Magister en Salud Pública \\ Facultad de Ciencias de La salud y los Alimentos \\ Universidad del Bio-Bio Avda. Andrés Bello s/n, Chillán, Chile \\ 422463350 klgo.mauricioarayasalgado@gmail.com \\ Doctor en Finanzas. Profesor asociado Facultad de Ciencias empresariales. Universidad del Bío-Bío \\ Av Andrés Bello s/n, Chillán, Chile 422463350 Email: alex@ubiobio.cl \\ (1) Artículo producto de investigación para tesis de Magíster en Programa de Magíster en Salud Pública, Universidad del Bío-Bío, Chile \\ (2) Autor de correspondencia \\ (3) No hay conflicto de interés por parte de los autores \\ Satisfacción laboral y clima organizacional en funcionarios de atención primaria de salud de una comuna en Chile
}

\section{Resumen}

Introducción: La satisfacción laboral y el clima organizacional son variables esenciales en la gestión de organizaciones. Su carencia contribuye a la aparición de efectos adversos como el ausentismo, rotación profesional y aparición de disfunciones o enfermedades, lo cual impacta en la organización, personal de salud y usuarios.

Objetivo: Determinar si existe relación entre la satisfacción laboral y el clima organizacional percibido por funcionarios de atención primaria de salud de una comuna en Chile.

Materiales y métodos: Estudio analítico de corte transversal en 110 funcionarios pertenecientes a dos Centros de Salud Familiar de una comuna en Chile. Se aplicó las escalas para medir satisfacción laboral y clima organizacional de Chiang et al. (2010). Para explorar correlaciones se utilizó prueba Rho de Spearmann.

Resultados: Ambos centros de salud presentaron un nivel medio de satisfacción laboral $(3,32 \pm 0,93)$ y clima organizacional $(3,45 \pm 0,83)$ de un máximo de 5 . La dimensión con promedio general más bajo de satisfacción laboral fue satisfacción por el ambiente físico $(2,80 \pm 0,93)$ y para clima organizacional fue reconocimiento $(3,12 \pm 0,77)$. En cuanto al promedio general más alto fue satisfacción con que realiza su trabajo $(3,56 \pm 0,87)$ y confianza $(3,66 \pm 0,88)$ respectivamente. La correlación entre los puntajes globales de ambas escalas fue estadísticamente significativa $0,636(\mathrm{p} \leq 0,001)$.

Conclusión: La percepción de un clima organizacional alto se relaciona con un mayor nivel de satisfacción laboral en los funcionarios de salud estudiados.

Palabras claves: Satisfacción laboral, Clima Organizacional, Personal de salud, Atención primaria de salud.

\section{Abstract}

\section{Introduction:}

Job satisfaction and organizational climate are essential variables in the management of organizations. Its lack contributes to the appearance of adverse effects such as absenteeism, professional rotation and appearance of dysfunctions or diseases, which impacts the organization, health personnel and users.

Objective: To determine if there is a relationship between job satisfaction and the organizational climate perceived by primary health care officials of a commune in Chile.

\section{Materials and Methods:}

Analytical cross-sectional study in 110 health staffbelonging to two Family Health Centers of a commune in Chile. Scales were applied to measure job satisfaction and organizational climate of Chiang et al. (2010). SPSS version 22 was used for the bivariate analysis of the data and Spearman's Rho correlation coefficient was applied.

Results: Both health centers presented an average level of job satisfaction $(3.32 \pm 0.93)$ and organizational climate $(3.45 \pm 0.83)$ out of a maximum of 5 . The dimension with the lowest general average of job satisfaction was satisfied by the physical environment $(2.80 \pm 0.93)$ and for organizational climate it was recognition $(3.12 \pm 0.77)$. Regarding the highest general average, he was satisfied with the fact that he performed his work $(3.56 \pm 0.87)$ and confidence $(3.66 \pm$ $0.88)$ respectively. The correlation between the global scores of both scales was statistically significant $0.636(\mathrm{p} \leq 0.001)$.

Conclusions: The perception of a high organizational climate is related to a higher level of job satisfaction in the health staff studied.

Keywords: Job satisfaction, Organizational climate, Health staff, Primary health care. 


\section{Introducción}

El aumento en la esperanza de vida y la alta prevalencia de enfermedades crónicas ha provocado una sobrecarga progresiva en el sistema de salud estatal y privado chileno [1], lo cual se traduce en que los profesionales que se desempeñan en el sector de la salud experimenten una gran demanda asistencial por servicios, con usuarios cada vez más informados sobre sus derechos y con expectativas cada vez más altas [2], lo que finalmente desafía las capacidades del actual sistema de salud [3]. Según un informe del Ministerio de Salud de Chile (MINSAL) en el año 2016 el universo nacional de funcionarios que se desempeñaban en la red pública de atención fue de 437.337 técnicos y profesionales. En cuanto al número de funcionarios contratados en los centros de atención primaria de salud municipalizados del país correspondía a 58.948 funcionarios. Por otro lado la densidad de médicos por 10.000 habitantes en el país fue de una tasa del 22,9 en 2016 siendo situado por debajo del promedio de países miembros de la OCDE [4]. Por lo que variables como: la sobredemanda cualitativa y cuantitativa, la sobrecarga, el trabajo bajo presión, cumplir con múltiples funciones de manera simultánea, y recursos humanos que no son proporcionales a la demanda de los usuarios, ha demostrado tener efectos perjudiciales sobre la salud de los trabajadores, tanto física como psicológicamente, afectando el trato al usuario, la calidad de atención, las relaciones interpersonales y favoreciendo la aparición de estrés laboral el cual puede ser precursor de ausentismo laboral y provocar diversas disfunciones y/o enfermedades [5-10]. Existen también antecedentes de la alta rotación de profesionales en atención primaria sobre todo de médicos que se encuentran insatisfechos en sus lugares de trabajo no logrando consolidarse en los equipos de salud y/o migrando al sistema privado, ya sea, por expectativas insatisfechas, falta de oportunidades de desarrollo profesional o incentivos inadecuados [6-8]. Como constructo, la satisfacción laboral engloba dos grandes áreas, la primera incluye los componentes emocionales hacia el trabajo y la segunda responde al balance entre resultados, expectativas y prestaciones que el trabajo ofrece realmente [11-12]. Existe evidencia que demuestra que aquellos funcionarios con altos niveles de satisfacción tienden a realizar mejor su trabajo, con mayor calidad, eficiencia y con un mejor desempeño [11-13].

No existe un consenso unánimemente aceptado en cuanto a su definición, pero podemos encontrar dos grandes orientaciones que nos pueden llevar a comprenderla: autores que la definen como una emoción, sentimiento o respuesta afectiva y otro grupo que la define como una actitud generalizada hacia el trabajo. Estas actitudes pueden ir referidas al trabajo en general o a facetas específicas del mismo. Finalmente ha de entenderse satisfacción laboral no como una conducta, sino como un conjunto de actitudes hacia los diversos aspectos del trabajo [11-12]. La satisfacción laboral se puede abordar desde diversas perspectivas que involucran condiciones físicas del medio ambiente laboral (iluminación, ventilación, temperatura, limpieza), hasta elementos como los valores y necesidades de los individuos, sus creencias, actitudes y motivación [14].

El concepto de clima organizacional conocido igualmente como (clima laboral) hace referencia a "los factores ambientales percibidos de manera consciente por las personas que trabajan en las organizaciones, los cuales se encuentran sujetos al control organizacional y que se traducen en normas y pautas de comportamiento"[11-12]. Se ha definido como las descripciones individuales del marco social que integra la persona [11]. El tratamiento del clima como percepción genérica de situaciones ha tenido la ventaja de permitir evaluaciones sumarias del contexto en investigaciones que de otra manera estarían focalizadas en gran parte a nivel individual [15-16]. Dos cualidades definidas y constantes del clima persisten en sus varias conceptualizaciones: es una percepción y es descriptiva [18-19]. Este concepto abarca desde características de la organización determinadas como percepciones, esquemas cognoscitivos y percepciones donde la persona y situación interactúan [20-22].

Atendiendo a lo anterior, el objetivo del presente estudio ha tenido por objetivo conocer la satisfacción laboral y el clima organizacional, y la relación existente entre estas dos variables organizacionales en funcionarios de atención primaria de salud de una comuna en Chile.

\section{Material y método}

Estudio analítico, correlacional de corte transversal. El universo de la muestra estuvo constituido por 136 funcionarios pertenecientes a dos centros de salud familiar de una comuna en Chile con corte al 31 de Julio del año 2017, a quienes se les aplicó un censo. Se incluyeron funcionarios con contrato vigente en calidad de titular y plazo fijo con más de 6 meses de antigüedad laboral y que accedieron a participar voluntariamente del estudio mediante consentimiento informado; excluyéndose al personal que se encontraba con licencia, permiso o en periodo de vacaciones.

\section{Instrumentos de recolección de datos}

La participación fue voluntaria, anónima y sin control de tiempo en el lugar de trabajo. Se entregó un sobre con las encuestas y un formulario con antecedentes sociodemográficos (sexo, edad) y laborales (antigüedad en el cargo, antigüedad en el centro de salud, centro de salud de desempeño, categoría funcionaria, tipo de contrato y jornada laboral) que los participantes debían completar.

Los datos de satisfacción laboral fueron obtenidos por medio de la escala de satisfacción laboral diseñada por los autores Meliá y Peiró [23] y adaptada por Chiang, Salazar, Huerta y Núñez [24] cuenta con una fiabilidad adecuada (alpha mayor a 0,9 ) actualmente contiene 44 ítems que conforman 10 dimensiones: Satisfacción por el trabajo en general, con el ambiente físico, con la forma en que realiza el trabajo, con las oportunidades de desarrollo, con la relación con el jefe, con la remuneración, con la autonomía, con el reconocimiento, con la sección o área y con la empresa [25]. Su formato de respuesta tipo Likert de 5 puntos abarca desde nada satisfecho a muy satisfecho. Los puntajes totales se calcularon a partir del promedio de sus dimensiones.

Por último se aplicó la escala para medir clima organizacional, basada en el instrumento de Koys \& Deccottis [26] adaptada 
por Chiang, Salazar, Huerta y Núñez [24] cuenta con una fiabilidad adecuada (alpha mayor a 0,9 ) contiene 42 ítems que conforman ocho dimensiones: autonomía, cohesión, confianza, presión, apoyo, reconocimiento, equidad e innovación. Cuenta con formato de respuesta tipo Likert de 5 puntos desde totalmente en desacuerdo a muy de acuerdo. Los puntajes totales se calcularon a partir del promedio de sus dimensiones.

Los datos fueron codificados con el programa Microsoft Excel y procesados con el programa estadístico SPSS versión 22 en español. Se aplicó prueba de Kolmogorov Smirnov para determinar la distribución de los datos y para evaluar la relación entre la satisfacción laboral el clima organizacional se utilizó el coeficiente de correlación Rho de Spearman ya que los datos no se ajustaron a la normalidad, se consideró un nivel de significancia de 0,05 .

\section{Resultados}

La tasa general de respuesta fue de un $81 \%$ correspondiente a 110 encuestas válidas, $68,18 \%$ de los participantes fueron mujeres y $31,81 \%$ hombres, con una edad promedio entre el rango de los 37-48 años y 26-36 años, respectivamente, con una antigüedad en el cargo actual entre los 5-9 años para mujeres y hombres. Con respecto a la antigüedad en el centro de salud mujeres y hombres se mantienen en el rango de 1 -9 años (Tabla $\mathrm{N}^{\circ} 1$ ).

TABLA N ${ }^{\circ}$. CARACTERIZACIÓN DE LA MUESTRA

\begin{tabular}{cccccccc} 
CENTRO DE SALUD & Universo & $\begin{array}{c}\text { Respuestas } \\
\text { válidas }\end{array}$ & $\begin{array}{c}\text { Hombre } \\
\mathrm{n}\end{array}$ & $\begin{array}{c}\text { Mujer } \\
\mathrm{n}\end{array}$ & $\begin{array}{c}\text { Tasa de } \\
\text { respuesta \% }\end{array}$ \\
\hline $\begin{array}{c}\text { Centro de salud } \\
\text { familiar N¹ }\end{array}$ & 70 & 52 & 19 & 36,5 & 33 & 63,5 & 74,3 \\
\hline $\begin{array}{c}\text { Centro de salud } \\
\text { familiar N² }\end{array}$ & 66 & 58 & 16 & 27,6 & 42 & 72,4 & 87,9 \\
\hline TOTAL & 136 & 110 & 35 & 31,8 & 75 & 68,2 & 80,8 \\
\hline
\end{tabular}

Fuente: Elaboración probia

La mayor proporción de participantes corresponde al estamento de los técnicos con un 41,81\% seguido de los profesionales no médicos con un 33,63\%. El 74,54\% se encuentra en calidad de contrato indefinido y el $90 \%$ con jornada completa de trabajo.

Como se puede observar en Tabla $\mathrm{N}^{\circ} 2$, la mayor proporción de hombres se encuentra satisfechos con un $51,43 \%$ y con respecto a las mujeres éstas se encuentran principalmente en un nivel medio de satisfacción con un 44\%. Al analizar la satisfacción laboral por rango de edad los funcionarios más jóvenes comprendidos entre los rangos de los 26 a 36 años y entre los 37 a 48 años presentaron un alto nivel de satisfacción con un $47,62 \%$ y un $44,74 \%$ respectivamente. En cambio los que se encuentran entre el rango de los 49 a 60 años perciben un nivel medio de satisfacción con un $57,69 \%$.
TABLA N ${ }^{\circ}$ 2. SATISFACCIÓN LABORAL

\begin{tabular}{|c|c|c|c|}
\hline \multirow{3}{*}{$\begin{array}{c}\text { Características } \\
\text { Sociodemográficas y } \\
\text { Laborales }\end{array}$} & \multicolumn{3}{|c|}{ SATISFACCIÓN LABORAL } \\
\hline & Nivel bajo & Nivel medio & Nivel alto \\
\hline & $\%$ & $\%$ & $\%$ \\
\hline \multicolumn{4}{|l|}{ SEXO } \\
\hline Hombre $(n=35)$ & 14,29 & 34,29 & 51,43 \\
\hline Mujer $(n=75)$ & 17,33 & 44 & 38,67 \\
\hline \multicolumn{4}{|l|}{ EDAD } \\
\hline 26 a 36 años $(n=42)$ & 19,05 & 33,33 & 47,62 \\
\hline 37 a 48 años $(n=38)$ & 18,42 & 36,84 & 44,74 \\
\hline 49 a 60 años $(n=26)$ & 11,54 & 57,69 & 30,77 \\
\hline más de 60 años $(n=4)$ & 0,0 & 50 & 50 \\
\hline \multicolumn{4}{|c|}{ ANTIGÜEDAD EN SU CARGO ACTUAL } \\
\hline menos de 1 año (n=7) & 28,57 & 14,29 & 57,14 \\
\hline 1 a 4 años $(n=24)$ & 29,17 & 33,33 & 37,5 \\
\hline 5 a 9 años $(n=36)$ & 13,89 & 52,78 & 33,33 \\
\hline 10 a 14 años $(n=19)$ & 5,26 & 42,11 & 52,63 \\
\hline más de 15 años $(n=24)$ & 12,5 & 37,5 & 50 \\
\hline \multicolumn{4}{|c|}{ ANTIGÜEDAD EN EL CENTRO DE SALUD } \\
\hline menos de 1 año $(n=4)$ & 0,0 & 0,0 & 100 \\
\hline 1 a 9 años $(n=56)$ & 23,21 & 41,07 & 35,71 \\
\hline 10 a 19 años $(n=29)$ & 3,45 & 51,72 & 44,83 \\
\hline 20 a 29 años (n=12) & 25 & 33,33 & 41,67 \\
\hline más de 30 años ( $n=9$ ) & 11,11 & 33,33 & 55,55 \\
\hline \multicolumn{4}{|l|}{ CATEGORÍA FUNCIONARIA } \\
\hline$A(n=14)$ & 14,28 & 42,86 & 42,86 \\
\hline$B(n=37)$ & 21,62 & 35,14 & 43,24 \\
\hline$C-D(n=46)$ & 13,04 & 43,48 & 43,48 \\
\hline$E(n=4)$ & 0,0 & 75 & 25 \\
\hline$F(n=9)$ & 22,23 & 33,33 & 44,44 \\
\hline \multicolumn{4}{|l|}{ TIPO DE CONTRATO } \\
\hline Indefinido $(n=82)$ & 14,63 & 42,68 & 42,68 \\
\hline Plazo fijo $(n=28)$ & 21,43 & 35,71 & 42,86 \\
\hline \multicolumn{4}{|l|}{ JORNADA LABORAL } \\
\hline Completa (44 h) (n=99) & 15,15 & 44,44 & 41,41 \\
\hline Parcial ( 33 o menos) $(n=11)$ & 27,27 & 9,09 & 54,55 \\
\hline
\end{tabular}

Fuente: Elaboración propia

En cuanto a la antigüedad en el cargo y en el centro de salud los resultados son bastante similares, debido a que no se abarcó una gran muestra y los grupos son bastante heterogéneos las valoraciones se agrupan en niveles medios y altos de satisfacción. En cuanto a la categoría funcionaria la mayor proporción de las categorías A (profesionales médicos), B (profesionales no médicos) y C-D (técnicos) se sitúan en un nivel medio y alto de satisfacción. La categoría E (administrativos) se encuentran en un nivel medio principalmente y finalmente la categoría F (auxiliares) en un nivel medio y alto. Para la variable tipo de contrato se logra visualizar que la mayoría de los funcionarios goza de contrato indefinido percibe niveles de satisfacción medio y alto con un $85,36 \%$. Finalmente quienes se desempeñan bajo una jornada completa de trabajo (99 funcionarios) presentan un nivel de percepción de satisfacción laboral de medio a alto con un $85,85 \%$.

En la Tabla $\mathrm{N}^{\circ} 3$, podemos apreciar que de los hombres participantes en el estudio un $60 \%$ percibe un nivel alto de clima organizacional. En cambio las mujeres perciben en mayor proporción un nivel medio con un 48\%, seguido muy de cerca por quienes perciben un nivel ato con un $45,33 \%$. 
TABLA N 3. CLIMA ORGANIZACIONAL

\begin{tabular}{|c|c|c|c|}
\hline \multirow{3}{*}{$\begin{array}{c}\text { Características } \\
\text { Sociodemográficas y } \\
\text { Laborales }\end{array}$} & \multicolumn{3}{|c|}{ CUMA ORGANIZACIONAL } \\
\hline & Nivel bajo & Nivel medio & Nivel alto \\
\hline & $\%$ & $\%$ & $\%$ \\
\hline \multicolumn{4}{|l|}{ SEXO } \\
\hline Hombre $(n=35)$ & 5,71 & 34,29 & 60 \\
\hline Mujer $(n=75)$ & 6,67 & 48 & 45,33 \\
\hline \multicolumn{4}{|l|}{ EDAD } \\
\hline 26 a 36 años $(n=42)$ & 2,38 & 35,71 & 61,90 \\
\hline 37 a 48 años $(n=38)$ & 7,89 & 50 & 42,11 \\
\hline 49 a 60 años $(n=26)$ & 11,54 & 50 & 38,46 \\
\hline más de 60 años $(n=4)$ & 0,0 & 75 & 25 \\
\hline \multicolumn{4}{|c|}{ ANTIGÜEDAD EN SU CARGO ACTUAL } \\
\hline menos de 1 año $(n=7)$ & 14,29 & 28,57 & 57,14 \\
\hline 1 a 4 años $(n=24)$ & 0,0 & 45,83 & 54,17 \\
\hline 5 a 9 años $(n=36)$ & 5,56 & 50 & 44,44 \\
\hline 10 a 14 años ( $n=19)$ & 0,0 & 42,11 & 57,89 \\
\hline más de 15 años ( $n=24)$ & 16,67 & 45,83 & 37,5 \\
\hline \multicolumn{4}{|c|}{ ANTIGÜEDAD EN EL CENTRO DE SALUD } \\
\hline menos de 1 año $(n=4)$ & 0,0 & 25 & 75 \\
\hline 1 a 9 años $(n=56)$ & 1,79 & 51,79 & 46,43 \\
\hline 10 a 19 años $(n=29)$ & 71 & 44,83 & 51,72 \\
\hline 20 a 29 años $(n=12)$ & 25 & 50 & 25 \\
\hline más de 30 años $(n=9)$ & 11,11 & 33,33 & 55,56 \\
\hline \multicolumn{4}{|l|}{ CATEGORÍA FUNCIONARIA } \\
\hline$A(n=14)$ & 0,0 & 28,57 & 71,43 \\
\hline$B(n=37)$ & 5,41 & 45,95 & 48,69 \\
\hline$C-D(n=46)$ & 8,70 & 43,48 & 47,83 \\
\hline$E(n=4)$ & 0,0 & 100 & 0,0 \\
\hline$F(n=9)$ & 11,11 & 55,56 & 33,33 \\
\hline \multicolumn{4}{|l|}{ TIPO DE CONTRATO } \\
\hline Indefinido $(n=82)$ & 8,54 & 45,12 & 46,34 \\
\hline Plazo fijo ( $n=28)$ & 0,0 & 42,86 & 57,14 \\
\hline \multicolumn{4}{|l|}{ JORNADA LABORAL } \\
\hline Completa (44 h) (n=99) & 5,05 & 48,48 & 46,46 \\
\hline Parcial (33 o menos) $(n=11)$ & 9,09 & 27,27 & 63,64 \\
\hline
\end{tabular}

Fuente: Elaboración propia

Del total estudiado quienes perciben un bajo nivel de clima organizacional con respecto a las variables sociodemográficas y laborales consideradas son una proporción bastante reducida. En cuanto a la edad, el rango etario más joven comprendido entre los 26 a 36 años percibe un clima organizacional alto con un $61,90 \%$. Los demás rangos desde los 37 hasta los mayores de 60 años se inclinan por una percepción media de clima organizacional. Para la variable antigüedad en el cargo los que se han desempeñado menos de un año y entre uno y cuatro años perciben un clima alto con un $57,14 \%$ y un $54,17 \%$, respectivamente. Aquellos con una antigüedad menor a un año y los que han trabajado más de treinta años en el centro de salud perciben un nivel alto de clima organizacional con un $75 \%$ y un $55,56 \%$, respectivamente. Con respecto a los estamentos, la categoría A (profesionales médicos) percibe un mayor nivel de clima organizacional con un $71,43 \%$. Por otro lado la categoría B (profesionales no médicos) y la categoría C-D (técnicos) se encuentran en un segmento medio alto de percepción. Para las categorías restantes E (administrativos) y F (auxiliares) la percepción se encuentra en un nivel medio con un $100 \%$ y $55,56 \%$, respectivamente. Por tipo de contrato, indefinidos y plazo fijo perciben un clima alto con un $46,34 \%$ y $57,14 \%$. Finalmente aquellos funcionarios con jornada completa se encuentran en un nivel medio con un $48,48 \%$ y los con jornada parcial mayoritariamente perciben un clima alto con un $63,64 \%$.

La relación más alta se presentó entre la dimensión satisfacción por el trabajo en general y confianza de clima organizacional $(0,660)$, innovación y satisfacción con el reconocimiento fue la segunda relación más alta con un valor de $(0,656)$ tabla $\mathrm{N}^{\circ} 4$. Para la dimensión presión de clima organizacional no se presentaron correlaciones estadísticamente significativas con las dimensiones de satisfacción laboral. Para la correlación entre los puntajes globales de ambas escalas de medición el resultado fue estadísticamente significativo con un valor de $0,636(\mathrm{p} \leq 0,001)$.

\section{Discusión}

En la organización estudiada se encontró que ambos centros de salud familiar presentaron en general un nivel de percepción medio de satisfacción laboral y clima organizacional. Ninguna dimensión de ambas variables reflejó un nivel bajo, todas oscilaron entre niveles medios y altos de percepción. Con respecto a la satisfacción laboral según características sociodemográficas y laborales se encontraron los siguientes resultados: Según sexo la mayor proporción de hombres se encontraron satisfechos $(51,43 \%)$ y con respecto a las mujeres éstas se encontraron en un nivel medio (44\%) de satisfacción laboral. Existe discrepancia al analizar la satisfacción laboral y edad de los funcionarios. Alonso [27] y Muñiz et al.[28] concluyen que a mayor edad se percibe una mayor satisfacción laboral, ya que con el tiempo las personas se encuentran más motivadas e implicadas en su trabajo.

En contraposición a esta postura se encuentra Takahara [29] quién plantea que con el pasar de los años las personas perciben que la carga laboral se acrecienta, por lo que a mayor edad disminuiría la satisfacción laboral. En el presente estudio los funcionarios más jóvenes situados entre el rango de los 26 a 36 años presentaron mayor nivel de satisfacción. Seguido del rango de los 37 a 48 años. Lo que se asemeja a lo reportado por Montoya et al. [30] donde el grupo que presenta mayor satisfacción corresponde a cuyas edades fluctúan entre los 35 y 49 años de edad, ya que se alcanza un mayor nivel de madurez el cual permite valorar el trabajo. Al analizar la satisfacción laboral por dimensiones de la totalidad de funcionarios del estudio destacan tres con un nivel alto de valoración: La primera es satisfacción con la forma en que realiza su trabajo $(3,56)$, la cual se relaciona con las oportunidades que posee el funcionario de realizar las cosas que le gustan y en las que se destaca, así como en la relación que tiene con su jefatura y las metas u objetivos que debe cumplir. En segundo y tercer lugar respectivamente destacan la satisfacción por la sección o área $(3,54)$ y satisfacción con la empresa $(3,52)$, lo cual muestra que los funcionarios se encuentran a gusto dentro de sus equipos de trabajo y en el centro de salud en el que se desempeñan. Esta tendencia se mantiene si se analiza por centro de salud. Las demás dimensiones se encasillan en un nivel medio de percepción, satisfacción con la autonomía $(3,47)$, con las oportunidades de desarrollo $(3,43)$, con el jefe $(3,36)$, con el trabajo en general $(3,31)$, con el reconocimiento $(3,13)$, con 
la remuneración $(3,02)$ y la dimensión con el nivel más bajo de este grupo corresponde a satisfacción con el ambiente físico con tan solo un $(2,80)$, lo cual incluye condiciones de iluminación, ventilación, temperatura, salubridad y espacio en que se desarrolla el trabajo. Cabe destacar que si se analiza por centro de salud, en ambos ésta es la dimensión con menor puntaje promedio, esta valoración se puede deber a que ambos centros de salud se encuentran en regulares condiciones y han tenido que optimizar su espacio para entregar cobertura a mayor cantidad de la población estimada en su construcción.

Con respecto a las dimensiones mejor valoradas del clima organizacional y que se encuentran en un nivel alto son: Confianza $(3,66)$, en la que destacan aspectos relacionados con la jefatura de sector a la que perciben como un superior de valores definidos y que cumple con los compromisos que adquiere con el trabajador, así como a la libertad para tratar temas sensibles sin temor a ser sancionados o perjudicados por expresarlos. En segundo lugar se encuentra autonomía $(3,59)$, en que el funcionario tiene cierto grado de libertad para organizar y decidir cómo ejecutar su trabajo. Apoyo $(3,52)$ respecto a la percepción de respaldo y tolerancia dentro del centro de salud lo que incluye el aprendizaje de los errores y la accesibilidad que otorga la jefatura al funcionario. Finalizando este grupo encontramos la dimensión de cohesión $(3,50)$, percepción sobre las relaciones interpersonales del equipo, como trabajan y se apoyan para lograr objetivos en común. Las siguientes dimensiones se encuentran en un nivel medio de valoración: Innovación $(3,45)$, en la que no existen muchas instancias en que se puedan generar nuevas formas de hacer las tareas. Esto se puede explicar en parte por los procesos de acreditación y certificación actuales en los que existen protocolos de atención establecidos para determinadas situaciones. La dimensión presión $(3,40)$, destaca como la que presenta menor desviación típica $(0,57)$ y se relaciona con la multiplicidad de tareas que se deben desarrollar en el lugar de trabajo y la relación con los usuarios, los cuales cada día se encuentran con más acceso a la información, conocen más sobre sus derechos y demandan mejores estándares de atención. Chiang et al. [21] al estudiar hospitales de alta y baja complejidad encontró que los funcionarios también asignaron niveles medios de satisfacción a la dimensión presión. Equidad $(3,39)$ y reconocimiento $(3,12)$ destacan con la valoración promedio más baja, la primera se refiere a la relación con el jefe de sector, si éste muestra favoritismos y otorga un trato justo a los funcionarios sin distinción y la segunda a la poca capacidad de las jefaturas para reconocer cuando el funcionario realiza bien una tarea y destacarlo. Si se analiza por centro de salud las dimensiones mejor y peor valoradas son similares.

Los puntajes globales de satisfacción laboral y clima organizacional presentaron una correlación estadísticamente significativa $(0,636)$. Lo que se condice con lo reportado en estudios de Chiang et al. [21], Cuadra y Veloso [19-20] y Montoya et al. [30]. Rodríguez [14] tras realizar una revisión de la literatura disponible plantea que las relaciones encontradas son de carácter moderado y oscilan entre 0,5 y 0,7 . La relación más alta se presentó entre la dimensión satisfacción por el trabajo en general y confianza de clima organizacional $(0,660)$. La dimensión presión de clima organizacional no presentó relación estadísticamente significativa con las dimensiones de satisfacción laboral, lo cual se asemeja a lo reportado por Montoya et al. [30] que en su estudio transversal correlacional del 2016 evaluó a 166 docentes y administrativos de una universidad estatal chilena y no encontró relación significativa para la dimensión presión. Del mismo modo Chiang et al. [21] en su estudio transversal correlacional del 2009 estudia hospitales de alta y baja complejidad y pese a trabajar con una muestra de 1.239 sujetos solo encuentra una relación significativa muy baja para la dimensión presión. Esto puede deberse a que cada contexto organizacional es diverso, por lo que cabe la posibilidad de encontrar discrepancias entre las diferentes realidades o contextos que se analicen [31].

\section{Conclusión}

La satisfacción laboral y el clima organizacional son herramientas de gestión ampliamente utilizadas en diversos contextos organizacionales. Su importancia radica en que permite elaborar diagnósticos de la situación laboral de sus trabajadores. A partir de los resultados obtenidos en la presente investigación, se encontró una relación estadísticamente significativa entre el clima organizacional y la satisfacción laboral $(0,636)$. Por lo tanto se puede concluir que la percepción de un clima organizacional alto se relaciona con un mayor nivel de satisfacción laboral. Se recomienda elaborar estrategias y planes enfocados a mejorar los niveles de valoración descritos, de tal manera que se cree una sincronía entre la misión y visión de la organización con las expectativas, necesidades, creencias, valores y actitudes de los funcionarios. La mejora de estos niveles de valoración puede impactar y favorecer directamente la productividad de los funcionarios, disminución del ausentismo y calidad de atención otorgada a los usuarios.

Dentro de las limitaciones, las percepciones de clima organizacional y satisfacción laboral se estudiaron por categoría funcionaria, lo cual si bien es un aporte, limita el conocimiento desagregado por quienes componen estas categorías (médicos, enfermeras, kinesiólogos, etc) y así realizar comparaciones con estudios similares. Para estudios posteriores en los que se desee evaluar esta relación se recomienda abarcar una mayor muestra como lo sugiere Mendoza [4] y Rodríguez et al. [11] que permita generalizar resultados y compararlos con otros estudios. Se recomienda también incorporar otras variables organizacionales como el liderazgo en el estudio de satisfacción laboral como Cuadra y Veloso [15] donde encuentran una correlación estadísticamente significativa entre liderazgo, satisfacción laboral y clima organizacional. 


\section{Referencias}

1. Ganga F, Piñones M, González D, Rebagliati F. Rol del Estado frente al envejecimiento de la población: el caso de Chile. Convergencia 2016; 23: 175-200.

2. González L, Guevara E, Morales G, Segura P, Luengo C. Relación de la satisfacción laboral con estilos de liderazgo en enfermeros de hospitales públicos, Santiago, Chile. Ciencia y Enf 2013; 19 (1): 11-21.

3. Bustamante-Ubilla MA, Grandón-Avendaño ML, Lapo-Maza MC. Caracterización del clima organizacional en hospitales de alta complejidad en Chile. Est Gerenc 2015; 31: 432-440.

4. Ministerio de Salud. Informe sobre brechas de personal de salud por servicio de salud. Santiago-Chile. 2017.

5. Mendoza R. Insatisfacción laboral como predictor del ausentismo en un hospital público. Rev Med Chile 2015; 143: 1028-1033.

6. Vilá M, Cruzate C, Orfilia F, Creixell J, González M, Davins J. Burnout y trabajo en equipo en los profesionales de atención primaria. Aten Primaria 2015; 47(1): 25-31.

7. Jiménez JP. Bienestar y satisfacción de los médicos con el ejercicio de su profesión en un sistema de salud en proceso de reforma. Rev Med Chile 2004; 132: 635-36.

8. Ríos F, Schonhaut L. Atención primaria de salud: factores de desmotivación y estabilidad laboral de médicos generales. Rev Clín Med Fam 2009; 2 (8): 378-385.

9. Bronkhorst B, Tummers L, Steijn B, Vijverberg D. Organizational climate and employee mental health outcomes: A systematic review of studies in health care organizations. Health Care Manag Rev 2015; 40(3): 254-271.

10. Fernández B, Paravic T. Nivel de satisfacción laboral en enfermeras de hospitales públicos y privados de la provincia de Concepción, Chile. Ciencia y Enf 2003; 9(2): 37-48.

11. Robbins S. Comportamiento organizacional. 8 ed. México: Prentice Hall, 1999.

12. Chiang Vega $M$, Martín Rodrigo $M$, Núñez Partido A. Relaciones entre el clima organizacional y la satisfacción laboral. Madrid: Universidad Pontificia Comillas, 2010.

13. Segura A. Clima organizacional: un modo eficaz para dirigir los servicios de salud. Av Enferm 2012; 30(1): 107-113.

14. Chiang M, Salazar M, Gómez M. Estilo de liderazgo y creencias organizacionales en la satisfacción laboral de la administración pública de Chile. ICADE 2014; 93: 103-122.

15. RodríguezA, Retamal M,Lizana J, Cornejo F. Clima y satisfacción laboral como predictores del desempeño: en una organización estatal chilena. Salud \& Sociedad 2011; 2 (2): 219-234.

16. Platis $\mathrm{CH}$, Reklitis $\mathrm{P}$, Zimeras $\mathrm{S}$. Relation between job satisfaction and job performance in healthcare services. PROCEDIA-Social and behavioral sciences 2015; 175; 480-487.
17. Schneider B, Ehrhart MG, Macey WH. Organizational climate and culture. Annu Rev Psychol 2013; 64: 361-88.

18. Schneider B, González-Romá V, Ostroff C, and West, Michael Alun. Organizational climate and culture:reflections on the history of the constructs in Journal of Applied Psychology. Journ of Appl Psychol 2017; 102(3):468-482.

19. Cuadra A, Veloso C. Liderazgo, clima y satisfacción laboral en las organizaciones. Rev Univers 2007; 2 (22): 40-56.

20. Cuadra A, Veloso C. Grado de supervisión como variable moderadora entre liderazgo y satisfacción, motivación y clima organizacional. Rev Chil Ing 2010; 18 (1): 15-25.

21. Chiang M, Salazar C, Martín M. Clima organizacional y satisfacción laboral. Una comparación entre hospitales públicos de alta y baja complejidad. Salud Trab 2011; 19 (1): 05-16.

22. Bustamante-Ubilla MA, Grandón-Avendaño ML, Lapo-Maza MC. Caracterización del clima organizacional en hospitales de alta complejidad en Chile. Est Gerenc 2015; 31: 432-440.

23. Meliá J. L, Peiró J. M. La medida de la satisfacción laboral en contextos organizacionales: El Cuestionario de Satisfacción S20/23. Psicologemas 1989; 5: 59-74.

24. Chiang M, Salazar C, Huerta P, Nuñez A. Clima organizacional y satisfacción laboral en organizaciones del sector estatal (instituciones públicas) desarrollo, adaptación y validación de instrumentos. Rev Univers 2008; 23 (2): 66-85.

25. Chiang M \& San Martín N. Análisis de la satisfacción y el desempeño laboral en los funcionarios de la Municipalidad de Talcahuano. Ciencia \& Trab 2015; 54: 159-165.

26. Koys D, DeCotiis T. Inductive Measures of Psychological Climate. Hum Relat 1991;44(3):265-285.

27. Alonso P. Estudio comparativo de la satisfacción laboral en el personal de administración. Rev Psichol Trab Organ 2008; 24(1):25-40.

28. Muñiz J, Peña-Suárez E, De la Roca $Y$, Fonseca-Pedrero E, Cabal A, García-Cueto E. Organizational climate in Spanish Public Health Services: Administrartion and Services Staff. Int Jour of Clin Health Psychol 2014; 14: 102-110.

29. Takahara R. Age-job satisfaction relantionship for japanese public school teachers: a ccomparison of techer's labor unión members and professional and technical employee members of prívate company labor unions. Sangyo Eiseigaku Zasshi 2014; 56(4): 91-101.

30. Montoya $\mathrm{P}$, Bello-Escamilla $\mathrm{N}$, Bermúdez N, Burgos F, Fuentealba M, Padilla A. Satisfacción laboral y su relación con el clima organizacional en funcionarios de una universidad estatal chilena. Ciencia \& Trab 2017; 7-13.

31. González-Burboa A, Manríquez C, Venegas M. Clima organizacional en una Dirección de Administración de Salud Municipal. Ciencia \& Trab 2014; 51: 152-157. 